\title{
Características del segmento de mercado norteamericano que visita Manabí - Ecuador.
}

\section{Characteristics of te North American market segment y hay Manabi- Ecuador visits.}

\begin{abstract}
Dra. Mabel Font Aranda. ${ }^{1}$, Ing. Liz Gabriela Mero López. ${ }^{2} \&$ MSc. Natacha Pahola de las Mercedes Díaz Rodríguez. ${ }^{3}$
\end{abstract}

\section{Resumen.}

El mercado norteamericano ha representado durante décadas uno de los dos primeros segmentos que visita Ecuador excepto 2017, esto condiciona la incidencia en la actividad turística. El objetivo del trabajo fue caracterizar el mercado estadounidense que visita la provincia Manabí para la incidencia en su satisfacción y regreso. Los pasos seguidos fueron: análisis de las políticas nacionales, caracterización de los territorios turísticos, recogida y procesamiento de información, descripción del turista norteamericano. Se emplearon métodos y técnicas como observación científica, revisión de documentos oficiales, estadística descriptiva, encuesta. El cuestionario se aplicó durante el mes de julio (mayor cantidad de llegadas durante 2016 y 2017). Se empleó un muestreo probabilístico aleatorio simple en los cantones Manta, Puerto López y de San Vicente la parroquia Canoa. Como resultado la mitad viaja en pareja o familia y un tercio solo, la mayor parte son masculinos, quince por ciento regresa a Manabí, más de la mitad evaluó buena la experiencia del viaje.

Palabras claves: Segmento de mercado, perfil del turista, turista norteamericano.

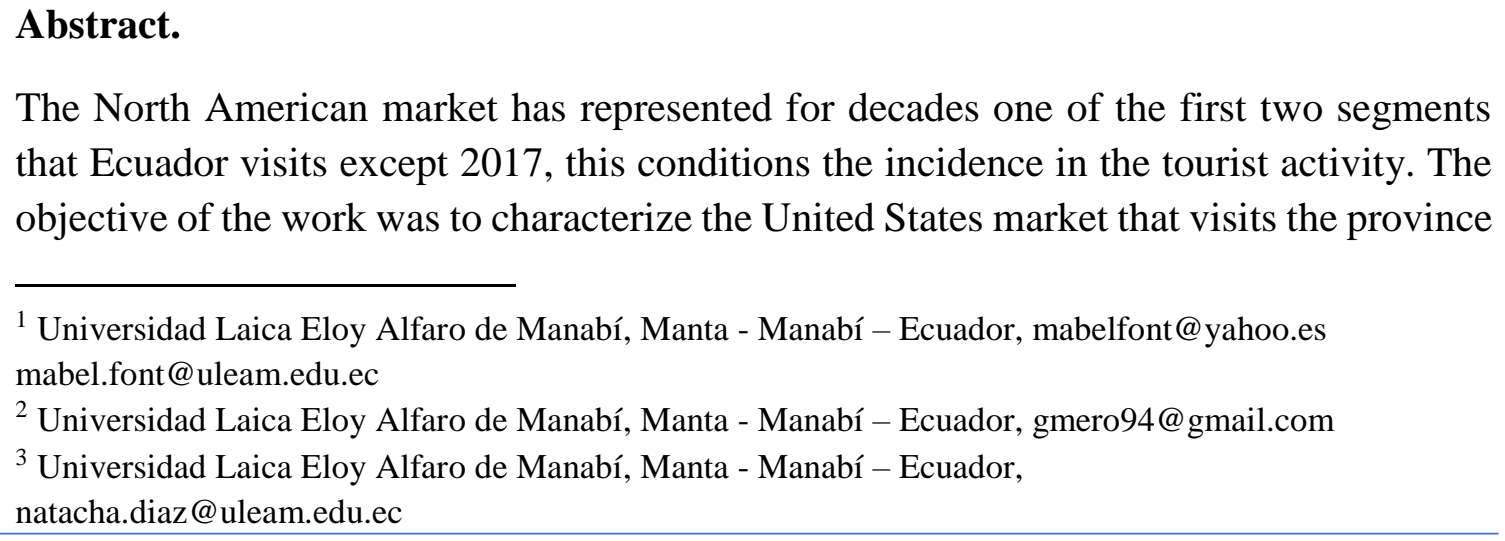

The North American market has represented for decades one of the first two segments that Ecuador visits except 2017, this conditions the incidence in the tourist activity. The objective of the work was to characterize the United States market that visits the province

\footnotetext{
${ }^{1}$ Universidad Laica Eloy Alfaro de Manabí, Manta - Manabí - Ecuador, mabelfont@ yahoo.es mabel.font@uleam.edu.ec

${ }^{2}$ Universidad Laica Eloy Alfaro de Manabí, Manta - Manabí - Ecuador, gmero94@ gmail.com

${ }^{3}$ Universidad Laica Eloy Alfaro de Manabí, Manta - Manabí - Ecuador, natacha.diaz@uleam.edu.ec
} 
of Manabí for its satisfaction and return. The steps taken were, analysis of national policies, characterization of tourist territories, collection and processing of information, description of the North American tourist. Methods and techniques were used as scientific observation, review of official documents, descriptive statistics, and surveys. The questionnaire was applied during the month of July (greater number of arrivals during 2016-2017). A simple random probabilistic sampling was used in the Manta, Puerto Lopez and San Vicente cantons. As a result, half travels as a couple or family and a third only, most are male, fifteen percent returns to Manabí, more than half said that the experience gained from the trip was good.

Keywords: Market segment, tourist profile, North American tourist.

\section{Introducción.}

El logro de la satisfacción del visitante y la adecuación de la oferta a sus gustos, solo es posible si conocemos su comportamiento, de tal forma, también es más rentable la empresa. Serra (2003), asigna importancia en turismo al comportamiento del consumidor, definiéndolo como el conjunto de actividades que lleva a cabo una persona desde que experimenta la necesidad de viajar hasta el momento en que efectúa la compra y utiliza los distintos servicios turísticos [1].

Para el conocimiento del mercado, una herramienta eficaz es la segmentación, porque de acuerdo a diferentes criterios comunes se agruparán las personas con intereses similares y en correspondencia será el producto y la oferta. Según Kotler (2011) un mercado es el conjunto de compradores actuales y potenciales de un producto. Estos compradores comparten una necesidad o deseo particular que se puede satisfacer mediante relaciones de intercambio. Son criterios de segmentación los deseos, recursos, ubicación geográfica, actitudes de compra y prácticas de compra, entre otros. Como los compradores no tienen necesidades y deseos únicos, cada comprador es potencialmente un mercado separado [2].

Resulta caduca la concepción de una oferta turística "todo para todos", es inoperante; la segmentación es una exigencia y herramienta factible, para la comercialización y expansión de los servicios turísticos Bigné (2000), Serra (2003), Kotler [et al.] (2011), Martínez-García, (2013) Ciribelli y Miquelito (2015) y Macías [et al.] (2017) [1, 2, 3, $4,5,6]$. La segmentación de mercados deja ver oportunidades y permite la satisfacción del consumidor, el conocimiento permanente de sus expectativas, la especialización en función de él y la proliferación de ventajas competitivas.

Acerca de los mercados emisores la Organización Mundial del Turismo (OMT, 2018) señaló que las llegadas de turistas internacionales se incrementaron un $7 \%$ en 2017 , siendo este el mayor crecimiento registrado en siete años. Respecto al turismo emisor medido por el gasto en turismo internacional, indica un resultado positivo en la mayor parte de los diez principales mercados emisores del mundo, los tres primeros representados por: China (+7\%), los Estados Unidos y Canadá (ambos $+9 \%$ ) [7].

En relación con lo antes plateado y en correspondencia con los datos del Ministerio de Turismo del Ecuador y el Instituto Nacional de Estadísticas y Censo (MINTUR - INEC. 2018), durante 16 años desde el 2000, el segmento norteamericano ha ocupado el primer 
o segundo lugar por visitantes, representado entre $24 \%$ y $15 \%$ del total de llegadas al territorio nacional. En 2017 dicho segmento representó 238625 llegadas, ocupando el tercer lugar por debajo de Colombia y Venezuela. No obstante, Estados Unidos es el primer país emisor de larga distancia. Del total de llegadas de turistas internacionales 22,2 $\%$ visitó la provincia Manabí [8].

El turista estadounidense, como se ha referido es de los mercados que tiene mayores gastos, y cambian de manera constante sus hábitos, en la búsqueda de información asociada a los avances tecnológicos, y las nuevas tendencias, siendo exigente en el mantenimiento y disfrutar su calidad de vida.

El análisis y caracterización del viajero estadounidense permitirá el conocimiento de su comportamiento, las motivaciones y tendencias de este segmento de turista, contribuyendo al desarrollo de mejores productos que inciten el regreso. Además, que se consoliden ofertas turísticas especializadas en el segmento de norteamericanos jovenadulto, adulto-jubilados y una prestación de servicios acorde a este segmento.

El planteamiento del problema científico se enmarca en la necesidad de conocer las expectativas, deseos, interés de los turistas norteamericanos que visitan la provincia de Manabí. La carencia o mala calidad de los servicios disminuyen el confort exigido por ellos y la satisfacción de sus necesidades, también el nivel de protección de los recursos turísticos natrales y culturales propios del litoral manabita.

Teniendo en cuenta lo expuesto, se formula el siguiente problema científico: ¿Cuáles son las características de los turistas norteamericanos que visitan la provincia Manabí, para incidir en la satisfacción de sus necesidades y expectativas en la prestación de diferentes ofertas, productos, servicios y experiencias?

Para responder al problema científico se plantea como objetivo general: Determinar el perfil del turista norteamericano que visita la provincia de Manabí para incidir en la satisfacción de sus necesidades y expectativas en la prestación de diferentes ofertas, productos, servicios y experiencias.

\section{Métodos.}

La investigación es aplicada descriptiva, tiene como campo de investigación el perfil del visitante norteamericano, en territorios turísticos representativos de Manabí, como: Manta, Puerto López y San Vicente (parroquia Canoa), la tabla 1 muestra los pasos seguidos y los métodos, técnicas y herramientas empleados.

Tabla 1. Procedimiento metodológico

\begin{tabular}{ll}
\multicolumn{1}{c}{ Pasos } & \multicolumn{1}{c}{ Métodos, Técnicas y Herramientas } \\
\hline 1- Políticas nacionales & Revisión de documentos oficiales \\
\hline $\begin{array}{l}\text { 2- Características de } \\
\text { los territorios turísticos } \\
\text { seleccionados }\end{array}$ & $\begin{array}{l}\text { Revisión de documentos oficiales } \\
\text { Cartográficos }\end{array}$ \\
\hline $\begin{array}{l}\text { 3- Recogida de } \\
\text { información }\end{array}$ & $\begin{array}{l}\text { Encuesta, aplicación de cuestionarios en el terreno según } \\
\text { muestreo } \\
\text { Trabajo de campo, observación científica, }\end{array}$ \\
\hline
\end{tabular}




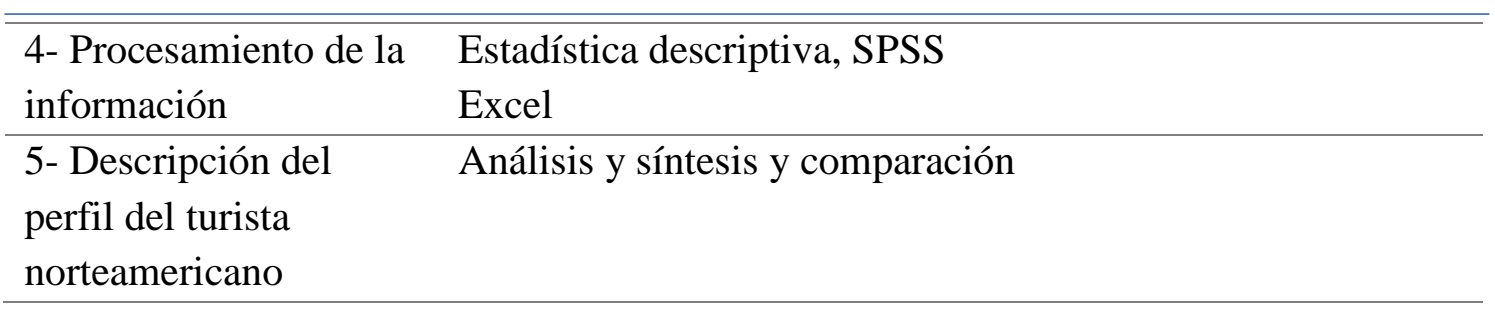

Fuente: Elaboración propia.

Para el análisis de las políticas nacionales que hilvanan con el tema de investigación y la caracterización geográfica cartográfica de los territorios turísticos, se emplea el método empírico de revisión de documentos oficiales, estos son: Plan Nacional de Desarrollo (2017-2021), PLANDETUR 2020, Plan de Desarrollo de Ordenamiento Territorial de Manta (2014-2019), Plan de Desarrollo de Ordenamiento Territorial de Puerto López (2015-2019), Plan de Desarrollo de Ordenamiento Territorial de San Vicente (2014-2019) $[9,10,11,12,13]$.

Se utiliza el cuestionario elaborado por especialistas de la ULEAM - MINTUR (2018) versión tercera "Encuesta de Turismo Nacional y Extranjero Manabí", que está estructurado en cuatro secciones con 21 preguntas: 1. Identificación del visitante, 2. Del viaje, 3. Gasto y 4. Destinos motivos y experiencias. En las interrogantes que miden satisfacción sobre calidad, precios, y cumplimiento de expectativas se emplea una escala de Likert de 5 opciones donde 1 es lo peor y 5 lo mejor. El resto se mueve entre 5 y 6 opciones que permiten describir al visitante.

El instrumento se aplica durante todo el mes de julio considerando el comportamiento de la estacionalidad de las llegadas de turistas norteamericanos en 2016 y 2017 MINTUR INEC (2018) y la disponibilidad de talento humano. La cifra de llegada de turistas norteamericanos fue de 33960 (2016) - 32438 (2017) en junio y 35862 (2016) - 31747 (2017) en julio, de ahí el valor más cercano fue 26066 (2016) - 24884 (2017) en diciembre.

Para seleccionar la muestra se consideró de partida 22,2\% (representó Manabí) del total de llegadas de norteamericanos a Ecuador en julio de 2017 (31 747), equivalente a 7048, de este se toma 75,11\% (5294 tamaño del universo) representativo de los cantones que reciben mayor cantidad de turistas internacionales: Manta, Puerto López y San Vicente. La muestra determinada fue de 266 en base a una probabilidad de ocurrencia $p=0,5$, un nivel de confianza de $95 \%$ y un error muestral de $6 \%$.

La elaboración y manejo de la base de datos se realiza con SPSS, mediante análisis, estadísticos descriptivos, frecuencias y tablas cruzadas, Excel favorece la representación gráfica.

\section{Resultados.}

Las políticas nacionales actuales están enfocadas hacia el turismo, la síntesis de los aspectos referidos en los documentos oficiales son: impulso de iniciativas para potenciar y desarrollar el sistema turístico, ejecución de eventos, con énfasis cultural para la 
atracción del turismo y el posicionamiento internacional, la reactivación de los sectores turísticos. En todos los documentos existe una declaración explícita acerca del rol del turismo, de la llegada de turistas nacionales y la movilidad del turismo interno, la implementación de estrategias que mejoren la calidad de vida del emprendedor o servidor y la experiencia del turista

La selección de los territorios turísticos objeto de estudio responde a MINTUR (2018), en el año 2017 la provincia Manabí representó el 22.2\% de las visitas internacionales, el total de visitantes para este año fue de 1,608 millones. Los cantones con mayor porcentaje fueron: Manta (31.15\%), Puerto López (27.11\%), San Vicente (16.85\%), que significó $75 \%$ del total de llegadas.

La provincia Manabí como muestra la figura 1, se ubica hacia el centro oeste del país, limitando con el Océano Pacífico. Siguiendo la orientación noreste - suroeste, colinda con las provincias Esmeraldas, Santo Domingo de los Tsáchilas Los Ríos, Guayas y Santa Elena.

A nivel provincial, Manabí se caracteriza por una gran riqueza de recursos y atractivos turísticos de carácter natural y cultural. la Agenda Zonal 4, describe todas las bondades que favorecen en este sentido al territorio, destacando la Ruta del Spondylus que bordea la costa, los variados paisajes y comunidades asentadas junto al litoral; favorables al crecimiento productivo y socioeconómico del turismo. El cantón Puerto López como Área Turística Protegida e incluyendo el Parque Nacional Machalilla, se distingue por la naturaleza y la accesibilidad de un muelle turístico para el avistamiento de las ballenas jorobadas, el buceo, esnórquel y las visitas a la isla de la Plata.

Las playas urbanas y rurales son notables en Manabí: Manta, San Jacinto, San Clemente, Bahía de Caráquez, San Vicente, Canoa entre otras abarcan más allá de los municipios turísticos., También los sitios arqueológicos de Cerro Jaboncillo, comuna Agua Blanca, entre otros y áreas protegidas bosques protectores, reservas, refugio y parque nacional). Los atractivos y ofertas citados articulan con diferentes rutas del Cacao y Café (agroturismo), Ruta del Encanto (balnearios de agua dulce), Ruta de los Abuelos (tradición oral y ecoturismo), Ruta del Sabor (gastronomía). Otras ofertas culturales se asocian a los museos y eventos de interés nacional e internacional.

En el proceso de recogida de información, se aplican de 5 a 10 encuestas, al inicio de mes la afluencia de turistas era mínima, pero fue incrementándose gradualmente, estimulada por el avistamiento de ballenas. El ambiente sicológico durante la aplicación del instrumento varía, aunque son personas cultas y educadas, con excelente comportamiento, en ocasiones se mostraron reacios a llenar el cuestionario. El carácter tolerable, videncia que las mujeres son mucho más amables que los hombres.

La aplicación de la encuesta tomo aproximadamente un tiempo de 5 a 7 minutos por persona, dependiendo de la disponibilidad del turista. Una parte de los turistas recomendó disminuir el número de preguntas del cuestionario, otros se mostraron solícitos y dispuestos.

El procesamiento de la información muestra los siguientes resultados, los turistas norteamericanos que llegan a Manabí, proceden de 17 estados, pero los que más se repiten son Florida, Texas, Nueva York y California, representan en cada caso entre 11 y $12 \%$. 
Un número reducido (8) tiene segunda residencia en Ecuador (figura 1), en Cuenca y Quito.

Respecto al género 56\% de los encuestados son masculinos y el resto femeninas. Los grupos etarios de mayor porcentaje corresponden a más de 52 años (45\%), como lo muestra la figura 2.

Figura 1. Mapa de Ecuador

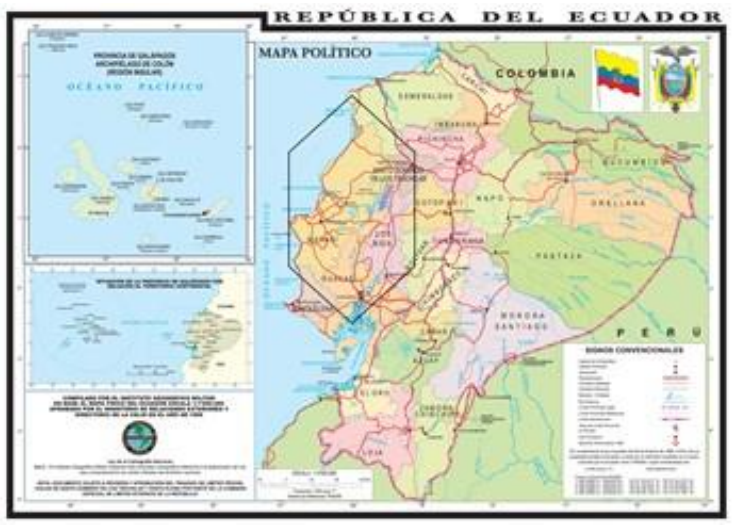

Figura 2. Composición de los grupos etarios.

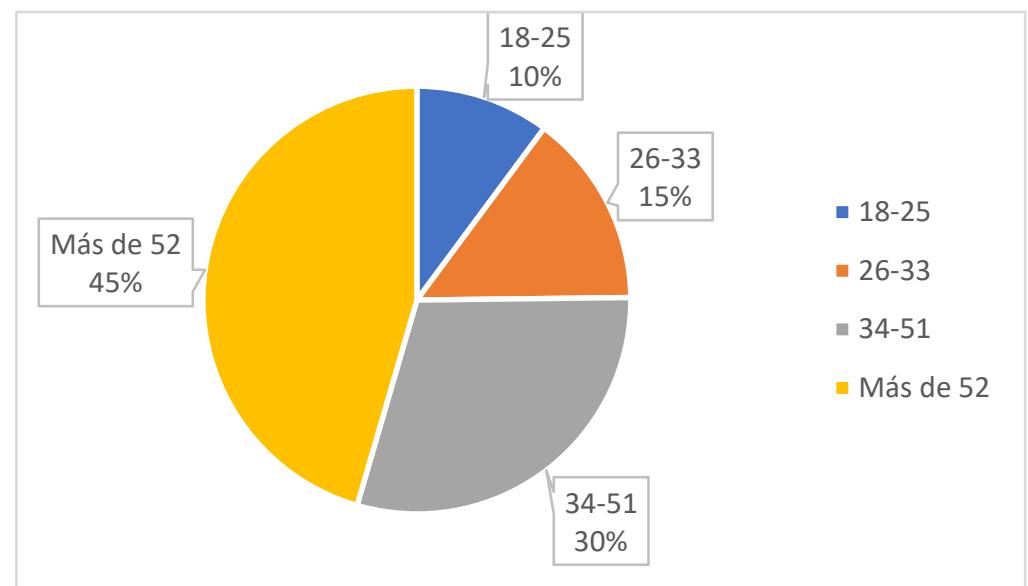

La figura indica que los servicios y ofertas deben tener en cuenta las necesidades de las personas de la tercera edad.

La mitad de los encuestados $50 \%$ tiene estado civil casado, mientras $29 \%$ está soltero, el cruce de información entre edad y estado civil muestra dos grupos uno corresponde a personas de más de 34 años, casados y otro con menos de 33 solteros, a esta configuración deben asociarse intereses diferentes.

Respecto a la situación laboral 43\% (114) se encuentra jubilado y 20\% (54) es empleado del sector privado, el resto de las categorías muestra comportamientos inferiores similares. Como es de predecir el grupo de más de 52 años incluye las personas jubiladas (54,5\%), también una parte reducida junto a los grupos etarios menores, tienen situación ocupacional de empleado, pero predominantemente privado. La situación laboral 
favorece la disponibilidad de recursos financieros para gastar y en el segmento de mayor edad tiempo para viajar.

A la pregunta sobre si es la primera vez que visita Manabí, 85\% (226) dice que es la primera vez, el resto inferior, pero no despreciable, ya ha estado antes en la provincia. Respecto a las noches pernoctadas la cifra superior es $33 \%$ una noche, el resto de las respuestas van desde 2 hasta 5 en cantidades relativamente equitativas, alrededor de 18 $\%$. Un valor extremo, pero interesante, representa 8 turistas que permanecieron 10 noches en los territorios turísticos, esta pregunta demuestra la heterogeneidad en las posibilidades y los gustos del turista norteamericano que visita Manabí.

Los visitantes estadounidenses viajan a Manabí en diferentes medios de transportes aéreo (33\%), público (28\%) y privado (39\%), como se muestra la opción terrestre privada, sobrepasa en 6 puntos al transporte aéreo. Las características de las vías de comunicación, la accesibilidad y la belleza del recorrido, puede ser un incentivo.

Un mes, es el tiempo que tiene mayor coincidencia en la preparación del viaje, 33\%, no obstante hay variedad de planificaciones desde una semana (25\%) hasta varios meses (24\%), no se evidencia un patrón único, algunos organizan la salida menos de una semana antes $(13 \%)$. El cruce de información tiempo de preparación con la edad, refleja que son los jóvenes los que se arriesgan a viajar aún menos de una semana. Las personas de los grupos etarios superiores preparan su salida con uno, varios meses (50\%) o hasta un año de antelación. Por otra parte, $62 \%$ de los encuestados utiliza Internet para panificar el viaje, y son algunos visitantes de edades más avanzadas, los que aún prefieren las agencias de viajes $(37 \%)$ y los folletos $(8,6 \%)$. De todos los grupos etarios, algunos también consultan a familiares y amigos (29\%).

Respecto a la estimación del gasto, se evidencia que las mayores estimaciones son: \$100200 (27\%), \$200 a 400 (32\%), mayor de \$400 (19\%). La valoración sobre los precios de los bienes y servicios consumidos (tabla 2), se muestra coincidencia significativa, $\mathrm{Ni}$ caro ni barato, es el que predomina en todos los casos, seguido de Barato.

Tabla 2. Calificación de los precios de los bienes y servicios adquiridos

\begin{tabular}{lccccccc}
\hline $\begin{array}{l}\text { Servicio/ } \\
\text { bienes }\end{array}$ & $\begin{array}{l}\text { Muy } \\
\text { Caro }\end{array}$ & Caro & $\begin{array}{l}\text { Ni barato, Barato } \\
\text { ni caro }\end{array}$ & $\begin{array}{l}\text { Muy } \\
\text { barato }\end{array}$ & $\begin{array}{l}\text { No } \\
\text { aplica }\end{array}$ & Total \\
\hline Alojamiento & 0 & 6 & 177 & 68 & 10 & 5 & 266 \\
\hline Alimentación & 0 & 6 & 171 & 70 & 17 & 2 & 266 \\
\hline Entretenimiento & 0 & 4 & 174 & 77 & 8 & 3 & 266 \\
\hline Compras & 4 & 8 & 162 & 66 & 13 & 13 & 266 \\
\hline Transporte & 1 & 8 & 128 & 66 & 21 & 42 & 266 \\
\hline
\end{tabular}

Fuente. Elaboración propia

Acerca de con quién realizó el viaje, $38 \%$ refiere solo y $50 \%$ en pareja o en familia, el predominio $60 \%$ viaja acompañado de una o dos personas más $60 \%$, y se alojan preferentemente en hoteles (56\%) y hostales (20\%). 
El motivo fundamental del viaje fue vacaciones y ocio, tratándose de destinos de sol y playa, es coherente que $89 \%$ responda afirmativamente la práctica de esta actividad, además $45 \%$ desarrolló actividades de turismo cultural y $34 \%$ de aventura y deporte, esta pregunta admitía varias respuestas.

Tabla 3. Motivo principal de su viaje.

\begin{tabular}{lcc} 
Motivos & Resultados & Porcentaje de 266 \\
\hline Vacaciones y ocio & 134 & $50 \%$ \\
\hline $\begin{array}{l}\text { Visita a familiares y } \\
\text { amigos }\end{array}$ & 66 & $25 \%$ \\
\hline $\begin{array}{l}\text { Educación / } \\
\text { entrenamiento }\end{array}$ & 19 & $7 \%$ \\
\hline Compras & 26 & $10 \%$ \\
\hline $\begin{array}{l}\text { Negocios y motivos } \\
\text { profesionales }\end{array}$ & 14 & $5 \%$ \\
\hline $\begin{array}{l}\text { Tratamientos de salud } \\
\text { / cuidados }\end{array}$ & 10 & $4 \%$ \\
\hline Otros & 30 & $11 \%$
\end{tabular}

Fuente. Elaboración propia.

Figura 3. Actividades que realizó.

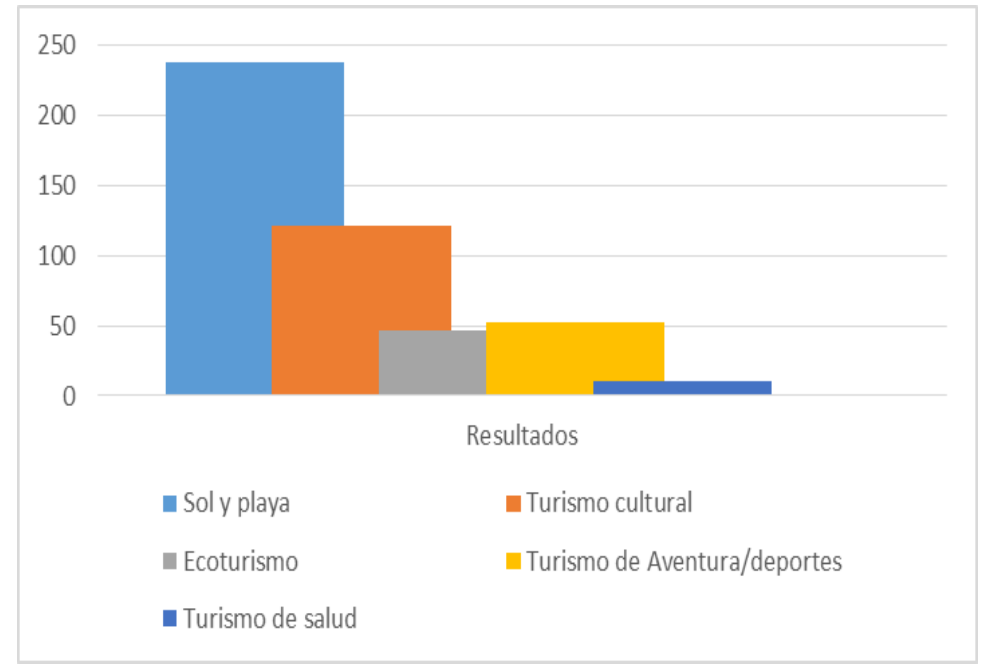

La calificación acerca de la calidad de los servicios consumidos muestra coincidencia en casi todos los rubros, las ponderaciones con mayores puntajes, corresponden a Buena y Muy Buena, aun así hay que vigilar las evaluaciones en Regular, y aceptar que existen falencias en la prestación de esos servicios.

Tabla 4. Calificación a la calidad de los servicios consumidos 


\begin{tabular}{|c|c|c|c|c|c|c|}
\hline \hline Servicios & Mala 2 & $\begin{array}{c}\text { Muy } \\
\text { mala 1 }\end{array}$ & Regular 3 & Buena 4 & $\begin{array}{c}\text { Muy } \\
\text { buena 5 }\end{array}$ & $\begin{array}{c}\text { No } \\
\text { aplica 6 }\end{array}$ \\
\hline Alojamiento & 1 & 0 & 39 & 128 & 91 & 9 \\
\hline $\begin{array}{c}\text { Comidas y } \\
\text { bebidas }\end{array}$ & 1 & 0 & 32 & 124 & 115 & 4 \\
\hline Recreación & 0 & 0 & 41 & 129 & 90 & 6 \\
\hline Bar y clubs & 0 & 0 & 37 & 126 & 83 & 20 \\
\hline Eventos & 0 & 0 & 38 & 57 & 35 & 136 \\
\hline Otro ¿Qué? & 0 & 0 & 5 & 61 & 18 & 182 \\
\hline
\end{tabular}

Fuente: Elaboración propia.

La pregunta final del cuestionario sirve de control a todas las interrogantes anteriores que miden satisfacción, 53\% de los encuestados evalúa Buena la experiencia del viaje a Manabí, y 28\% Muy Buena. En sentido opuesto $18 \%$ siente que las experiencias vividas han quedado por debajo de sus expectativas.

\section{Discusión.}

Dado que un porcentaje importante de turistas norteamericanos que visita Manabí, corresponde a los grupos etarios mayores; manifiesta características afines, prepara su viaje con tiempo, se informa por diferentes vías incluyendo Internet, agencias de viajes, folletos, otros y exige calidad en la atención, estos aspectos son referenciados por Martínez-García (2013) [5] y MINTUR, (2018) muestra estadísticas similares en los grupos etarios, diferenciado una parte de jóvenes de menos de 30 años y otra de la tercera edad o adultos, de aquí se derivan diferencias en la mayoría de os aspectos.

Se coincide a diferencia de 1\%. con Macías [et al.] (2017), sólo el 14\% de los encuestados tenían una experiencia previa en el país como visitante y el $86 \%$ venía a Ecuador por primera vez [5].

Los norteamericanos también buscaban opciones de alimentación más saludables, y sus menús no ofrecían demasiadas alternativas, plantea Kotler (2011) y continúa diciendo que preocupados por su salud, comen menos en restaurantes de comida rápida [2]. En Manabí la gastronomía es peculiar, sana y natural, se corrobora con 90\% de encuestados que califican este rubro bueno y muy bueno.

El segmento estadounidense gasta durante su estancia en el destino, hasta más de 400 dólares demostraron Plaza Obando (2014) y Matovelle y Báez, (2018) [14, 15]. En el presente estudio son los que más gastan en Manabí el porcentaje más representativo de \$200 -400, esto guarda relación con la valoración de los precios como Ni barato ni caro y Barato.

\section{Conclusiones.}

Los aspectos teóricos/metodológicos, facilitan el desarrollo de la investigación y la caracterización del turista estadounidense que visita la provincia Manabí, permitiendo cumplir el objetivo propuesto y su contribución a la solución del problema científico. 
El turista norteamericano que visita Manabí se caracteriza por viajar predominantemente en pareja o en familia, de o tres personas, si es parte de los más jóvenes prepara su viaje días antes a través de Internet, si pertenece a los de mayor edad, utiliza agencias de viajes o folletos y puede comenzar a preparar el viaje varios meses antes.

$>$ El viajero de Estados Unidos gasta en Ecuador; es un mercado histórico que en su estadía deja ganancias a las comunidades y prestadores de servicios, en igual dimensión exige, calidad en su experiencia.

La determinación del perfil del turista norteamericano facilita la comprensión de sus gustos y exigencia, y debe sustentar el diseño de ofertas y experiencias a tono con sus expectativas, estimulando el incremento de las llegadas de este segmento de mercado.

\section{Referencias Bibliográficas.}

SERRA CANTALlOPS, A. Marketing Turístico. Editorial Pirámide, Madrid, 2003. ISBN 84-368-1653-6.

KOTLER PHILIP, [et al.]. Marketing Turístico, 5. Ed. Pearson Educación, S.A., Madrid, 2011. ISBN: 978-84-8322-808-1.

BIGNÉ, E. J. [et al.] Marketing de Destinos Turísticos: análisis y estrategias de desarrollo. Editorial ESIC 2000. ISBN.

MARTÍNEZ-GARCIA, E. El turista sénior en Europa: actualidad y futuro Revista Iberoamericana de Turismo - RITUR, Penedo, vol. 3, n.1, p. 38-56, 2013. http://www.seer.ufal.br/index.php/ritur.

MACÍAS CLAVIJO T.A. [et al.] Percepciones de Ecuador como Destino según el Turista que Viaja desde Guayaquil. PODIUM No. 31, Guayaquil, Junio 2017, pp. 69-86 Universidad Espíritu Santo - UEES. ISSN: 1390 - 5473. Recuperado de: http://revistas.uees.edu.ec/index.php/Podium/article/view/99/91

CIRIBELLI, J. P. Y MIQUELITO S. La segmentación del mercado por el criterio psicográfico. Revista Científica "Visión de Futuro" Año 12, Volumen No19, No 1 , Enero - Junio 2015 - Pág. 33 - 50

OMT. Comisión de la OMT para las Américas 63ª reunión Asunción (Paraguay), 13 de abril de 2018 Punto 3 del orden del día provisional CAM/63/3 Madrid, 3 de abril de 2018 Original: Inglés Organización Mundial del Turismo (UNWTO) Organismo especializado de las Naciones Unidas. Madrid (España). Recuperado de: http://cf.cdn.unwto.org/sites/all/files/pdf/3._informe_del_sg.pdf 
MINTUR - INEC. Anuario de entradas y salidas internacionales al Ecuador 2000-2017.

Dirección de Inteligencia de Mercados. Coordinación General de Estadísticas e Investigación. $\quad 2018 . \quad$ Recuperado de: http://servicios.turismo.gob.ec/index.php/turismo-cifras/2018-09-19-17-0151/movimientos-internacionales Consultado: Julio de 2018. Quito.

SENPLADES. Plan Nacional de Desarrollo (2017-2021). 2017. Edición - Quito Ecuador.

MINTUR. PLANDETUR, 2020. Plan Estratégico de Desarrollo de Turismo Sostenible. 2007. Quito - Ecuador.

GAD - MANTA (Gobierno Autónomo Descentralizado del Cantón Manta). 2014. Plan de Desarrollo y Ordenamiento Territorial 2014 - 2019. Manta. Manabí.

GAD - PUERTO LÓPEZ (Gobierno Autónomo Descentralizado del Cantón Puerto López). Plan de Desarrollo y Ordenamiento Territorial (2015 - 2019). 2015. Puerto López. Manabí.

GAD - San Vicente (Gobierno Autónomo Descentralizado del Cantón San Vicente). Plan de Desarrollo y Ordenamiento Territorial (2015 - 2019). 2015. San Vicente. Manabí.

MATOVELlE, P. A. T; BAEZ, S. Medición de la experiencia del turista en Quito. Rev. Bras. Pesq. Tur. São Paulo, 12(1), pp. 102-132, jan./abr. 2018. Recuperado de: http://www.scielo.br/readcube/epdf.

PLAZA OBANDO M. C. Estudio de factibilidad para la creación de un hostal residencia, en la parroquia de Canoa, provincia de Manabí-Ecuador" Trabajo de titulación presentado en conformidad a los requisitos establecidos para optar por el título de Ingeniera en Administración de Empresas Turísticas y Hoteleras. 2014. Facultad De Ciencias Económicas Y Administrativas Escuela de Hotelería y Turismo.

\section{UL Ciencia}




\section{Para citar el artículo indexado.}

Font M., Mero L. \& Díaz N. (2019) Características del segmento de mercado norteamericano que visita Manabí - Ecuador. Revista electrónica Explorador Digital 3(2), 36-47. Recuperado desde:

http://cienciadigital.org/revistacienciadigital2/index.php/exploradordigital/article/view/418/9 $\underline{53}$

\section{Ciencia \\ Digital \\ Editorial}

El artículo que se publica es de exclusiva responsabilidad de los autores y no necesariamente reflejan el pensamiento de la Revista Explorador Digital.

El articulo queda en propiedad de la revista y, por tanto, su publicación parcial y/o total en otro medio tiene que ser autorizado por el director o editor de la Revista Explorador Digital.
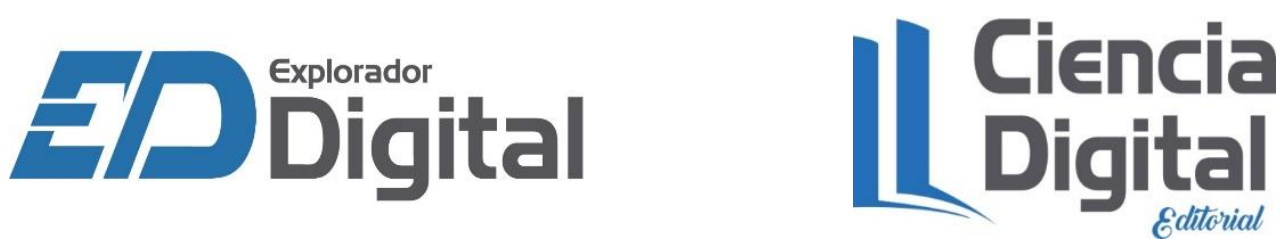